BULL. AUSTRAL. MATH. SOC.

VOL. $4(1971), 367-387$.

\title{
On the Krull Galois theory for non-algebraic extension fields
}

\section{T. Soundararajan and K. Venkatachaliengar}

\begin{abstract}
The Krull Galois theory for infinite separable normal extensions is generalized in this note to non-algebraic extensions. For any extension field $E$ of a field $K$ it is shown that the Galois group $G$ can be given a translation invariant topology such that the closed subgroups are precisely the subgroups that figure in a Galois correspondence. For extension fields $E / K$ such that $E / K$ is of finite transcendence degree and such that $E$ is Galois over each intermediate field the topology turns out to be compact and we have a Galois correspondence in the Krull fashion. For infinite transcendence degree extensions the Galois correspondence remains but compactness is lost. The topology coincides with the Krull topology in the case of algebraic extensions. Further properties of the topology are also studied.
\end{abstract}

Classical Galois Theory asserts that if $E$ is a finite separable normal extension of a field $K$ then there is a one-one Galois correspondence between all intermediate fields of $E / K$ and all subgroups of the Galois group of $E / K$. Krull [3] generalized this by showing that if $E$ is any separable normal extension of $K$, then a topology (Krull topology) can be put on the Galois group $G$ of $E / K$ so that there is a one-one Galois correspondence between all the intermediate fields of $E / K$ and all the topologically closed subgroups of $G$. With the Krull topology

Received 2 December 1970. The authors wish to express their best thanks to Professor M. Venkataraman for his encouragement in the preparation of this paper. 
$G$ becomes a compact Hausdorff topological group. When $E / K$ is finite the Krull theory gives back the classical Galois theory.

The question arises whether we could consider a not necessarily algebraic extension $E / K$, have a suitable topology on the Galois group and still have a one-one Galois correspondence between all intermediate fields and all topologically closed subgroups. The first necessary condition to have all these is, we must restrict ourselves to Dedekind extensions, that is, extensions $E / K$ such that $E$ is Galois over each intermediate field of $E / K$ (for example, the field of complex numbers over the rationals).

If $E / K$ is a Dedekind extension and we try to have a one-one Galois correspondence between all intermediate fields and all topologically closed subgroups of $G$ for some topology then [9] shows that unless the topology is very weak we will be forced to consider algebraic separable normal extensions only: while [10] shows that there are topologies, though very weak, which permit a topological Galois correspondence. But these do not coincide with $\mathrm{Krull}$ topology when we consider algebraic extensions.

In this note we generalize Krull Galois theory by proving the following results:

(I) If $E / K$ is any Dedekind extension of finite transcendence degree then the Galois group $G$ of $E / K$ can be given a topology $J$ such that

(a) $J$ is compact:

(b) for $(G, J)$ translations and inverse are homeomorphisms:

(c) there exists a one-one Galois correspondence between all intermediate fields of $E / K$ and all J-closed subgroups of $G$. (Theorems 2.8 and 2.10. )

(2) If $E / K$ is any Dedekind extension of infinite transcendence degree then there need not exist a topology on the Galois group $G$ satisfying conditions (a) to (c) of ( 1 ), but there will always exist a topology $J$ satisfying the conditions (b) and $(c)$. (Theorems 2.8 and 2.11.)

(3) When $E / K$ is algebraic the topology $J$ coincides with the Krull topology and conversely. (Theorem 2.9.)

(4) If $E / K$ is any Galois extension with Galois group $G$ then we 
know that there is a one-one Galois correspondence between some intermediate fields (Galois closed intermediate fields) and some subgroups of $G$ (Galois closed subgroups) of $G$. [5] and [10] give topological characterizations of these Galois closed fields and subgroups respectively. In $\$ 3$ we show that we can place a topology $J$ on $G$ such that Galois closed subgroups are precisely the J-closed subgroups and such that for $(G, J)$ translations and inverse are homeomorphisms. (Theorem 3.1.)

In $\$ 4$ we consider properties of the topology $J$ and especially try to answer when it is compact.

$$
1 .
$$

In this section, we prove a group theoretical lemma needed for $\S 2$ and $\S 3$.

LEMMA 1.1*. Let $G$ be a group and $G_{1}, G_{2}, \ldots, G_{p}$ be a finite number of subgroups of $G$. Let $H$ be another subgroup of $G$. Further, Let $H$ be contained in a set union of a finite number of (left) cosets of these $G_{i}$, with cosets of $G_{i}$ being needed for each $i$. Then $H$ is contained in a set union of a finite number of cosets of $G_{1} \cap \ldots \cap G_{p}$.

Proof. Let

$$
\begin{aligned}
& H \subset a_{11} G_{1} \cup a_{12} G_{1} \cup \ldots \cup a_{1 r_{1}} G_{1} \cup a_{21} G_{2} \cup a_{22} G_{2} \cup \ldots \cup a_{2 r_{2}} G_{2} \cup \ldots \\
& \quad \cup a_{i 1} G_{i} \cup a_{i 2} G_{i} \cup \ldots \cup a_{i r_{i}} G_{i} \cup \ldots \cup a_{r 1} G_{r} \cup a_{r 2} G_{r} \cup \ldots \cup a_{r r_{r}} G_{r} .
\end{aligned}
$$

If $i_{1}, i_{2}, \ldots, i_{s}$ are elements from $1,2, \ldots, r$ we let

$G_{i_{1} i_{2} \ldots i_{s}}=G_{i_{1}} \cap \ldots \cap G_{i_{s}}$. We can suppose that each coset contains an element of $H$ not belonging to any other coset (otherwise that particular coset can be dropped out of the picture). Since a coset can be represented by any of its elements we can suppose that $a_{i j}$ for each $i$ and $j$ belongs to $H$ and belongs only to the coset $a_{i j} G_{i}$. Consider the cosets of $G_{1}$. Since $H$ is not covered by these cosets of $G_{1}$, there is an element $\alpha$ of $H$ not belonging to any of the cosets of $G_{1}$.

\footnotetext{
* This can also be easily deduced from a theorem of B.H. Neumann [4].
} 
We can choose this $\alpha$ to belong to only one coset. Consider now particularly the coset $a_{11} G_{1}$. Here $a_{11} \in H$ and $a_{11}$ does not belong to any other coset. $\alpha=p a_{11}, p \in H$ as $H$ is a group. Let us now consider $\left\{p a_{11} g_{1} \mid a_{11} g_{1} \in H, g_{1} \in G_{1}\right\}$.

Let $a_{11} g_{1} \in H, g_{1} \in G_{1}$. Then $p a_{11} g_{1} \in H$ since $p \in H$ and $H$ is a subgroup. We claim that $p a_{11} g_{1}$ does not belong to any coset of $G_{1}$. For if $p a_{11} g_{1} \in a_{1 j} G_{1}, I \leq j \leq r_{1}$ then $p a_{11} g_{1}=a_{1 j} g_{2}$, $g_{2} \in G_{1}$. Hence $p a_{11}=a_{1 j} g_{2} g_{1}^{-1}$ so that $\alpha=p a_{11} \in a_{1 j} G_{1}$. This is a contradiction since $\alpha$ does not belong to any of the cosets of $G_{1}$. Hence for some $i \neq 1, p a_{11} g_{1} \in a_{i j} G_{i}$. Suppose also that $p a_{11} h_{1} \in a_{i j} G_{i}$ with $a_{11} h_{1} \in H, h_{1} \in G_{1}$. Then we have

$$
\begin{array}{ll}
p a_{11} g_{1}=a_{i j} g_{i}, & g_{i} \in G_{i}, \\
p a_{11} h_{1}=a_{i j} h_{i}, & h_{i} \in G_{i},
\end{array}
$$

so that $\left(p a_{11} g_{1}\right)^{-1}\left(p a_{11} h_{1}\right)=\left(a_{i j} g_{i}\right)^{-1}\left(a_{i j} h_{i}\right)$ that is, $g_{1}^{-1} h_{1}=g_{i}^{-1} h_{i}$.

But $g_{i}^{-1} h_{i} \in G_{i}$ and $g_{1}^{-1} h_{1} \in G_{1}$. Hence $g_{1}^{-1} h_{1} \in G_{1 i}$. Hence $h_{1} \in g_{1} G_{1 i}$. Then $a_{11} h_{1} \in a_{11} g_{1} G_{1 i}$. If no other $p a_{11} h_{1} \in a_{i j} G_{i}$ for $a_{11} h_{1} \in H$ and $h_{1} \in G_{1}$ we easily see that $a_{11} g_{1} \in a_{11} g_{1} G_{1 i}$.

Thus as far as elements of $H$ are concerned the coset $a_{11} G_{1}$ can be replaced by finitely many cosets $a_{11} g_{1} G_{1 i}$ with $i \neq 1$ and one of them will have the form $a_{11} G_{1 j}$ with $p a_{11}$ in a coset of $G_{j}$. Since $p a_{11}$ belongs to only one coset, it cannot happen here that for some $a_{11} h_{1} G_{1 t}$, $h_{1} \in G_{t}$. For otherwise then $h_{1} \in G_{1 t}$. But by our choice $p a_{11} h_{1} \in a_{t l} G_{t}$. Then $p a_{11} \in a_{t l} G_{t}$. This would imply that $t=j$, so that $h_{1} \in G_{j}, a_{11} h_{1} G_{1 j}=a_{11} G_{1 j}$. This is a repeated coset which we could suppose has been written only once. A similar thing can be done for 
the other cosets of $G_{1}$, and then we could do a similar thing for the cosets of $G_{i}$ for $i=2,3, \ldots, r$. Then find $H \subset \cup b_{i j l} G_{i j} ; \quad \imath$ suitably varying, with each $b_{i j l}$ being of the form $a_{i j} g_{i}$ where $g_{i} \in G_{i}$.

We now successively omit some of the cosets if they are superfluous as far as $H$ is concerned. We assert that when we have come to a minimal covering, for each $a_{i j}$ there is some coset $a_{i j} G_{i k}$. For otherwise $a_{i j} \in a_{l m} g_{l} G_{l t}, g_{\eta} \in G_{\eta}, g_{\eta} \notin G_{l t}$. This implies that $a_{i j} \in a_{2 m} g_{\eta} G_{l}$ since $G_{Z t} \subset G_{Z}$ and so $a_{i j} \in a_{Z m} G_{Z}$. This means that $i=l$ and $j=m$. Now $a_{i j} \in a_{i j} g_{i} G_{i t}$. This implies that $g_{i} \in G_{i t}$ which is not possible by our choice of the $g_{i}$.

If now we have $G_{i j}=G_{k l}$ we can write both as $G_{i j k l}$. If further $G_{k l}=G_{p q}$ we can write all the three as $G_{i j k l p q}$ and so on. Hence we have again a situation where $H$ is contained in a finite set union of cosets of subgroups of $G$ and here each subgroup involves at least two of the indices $1,2, \ldots, r$. Now we can repeat the process done earlier and proceed. We again get $H$ contained in a finite set union of sugroups, and each subgroup involves at least three distinct indices now. Each time in the process if a coset involves a subgroup equal to $G_{12 \ldots r}$, we need not apply the process to that coset.

Proceeding thus in a finite number of steps we get that $H$ is contained in a set union of a finite number of cosets of $G_{12 \ldots r}$. This proves the lemma.

\section{Extension of the Krull Galois theory}

DEFINITION 2.1. Let $E$ be a field and $F$ a subfield. We say $E$ is Galois over $F$ or $E$ is a Galois extension of $F$ if $F$ is the fixed field of the group of all automorphisms of $E$ over $F$; that is given any element $x \in E, x \notin F$ there is an automorphism of $E$ which fixes each element of $F$ but which moves $x$. The group of all automorphisms of $E$ over $F$ we call the Galois group of $E$ over $F$. 
DEFINITION 2.2. Let $E$ be an extension field of a field $K$. We say $E$ is a Dedekind extension of $K$ if for each intermediate field $F$ of $E / K, E$ is Galois over $F$; that is if $F$ is a field such that $K \subseteq E \subseteq E$ then $E$ is a Galois extension of $F$.

DEFINITION 2.3. Let $E$ be a field and $H$ a group of automorphisms of $E$. By the fixed field of $H$ we mean the set

$$
I(H)=\{x \in E \mid s(x)=x \text { for each } s \in H\} .
$$

DEFINITION 2.4. Let $E$ be an extension field of a field $K$ and let $E$ be Galois over $K$. Let $G$ be the Galois group of $E$ over $K$. Let $H$ be a subgroup of $G$. We say $H$ is Galois closed if $H=\{\sigma \in G \mid \sigma(x)=x$ for every $x \in I(H)$ where $I(H)$ is the fixed field of $H$ \}.

DEFINITION 2.5. Let $E$ be an extension field of a field $K$ and let $E$ be Galois over $K$. Let $G$ be the Galois group of $E$ over $K$. If $x$ is an element of $E$ we write $G(x)=\{\sigma \in G \mid \sigma(x) \neq x\}$. If $s$ and $t$ are elements of $G$, we write $s G(x) t=\{s \sigma t \mid \sigma \in G(x)\}$. Let $F$ be the collection of all sets $s G(x) t, s$ and $t$ are elements of $G$ and $x$ an element of $E$. If $G(x)=\emptyset$ we let $s G(x) t$ also be $\emptyset$.

Then the collection $F$ constitutes a sub-base of open sets for a topology $J$ on $G$. A base for this topology $J$ consists of sets of the form

$$
s_{1} G\left(x_{1}\right) t_{1} \cap s_{2} G\left(x_{2}\right) t_{2} \cap \ldots \cap s_{n} G\left(s_{n}\right) t_{n} .
$$

Through this paper $J$ will refer to this topology only.

PROPOSITION 2.6. Let $E$ be a Galois extension of a field $K$ and let $G$ be the Galois group of $E$ over $K$. Then for any $x \in E$ and $s \in G$ we have

(1) $\{G(x)\}^{-1}=G(x)$ if $G(x) \neq \varnothing$,

(2) $s G(x) s^{-1}=G(s(x))$.

Proof. (1) follows from the fact that if $\sigma \in G$ and $x \in E$ then $\sigma(x) \neq x$ if and only if $\sigma^{-1}(x) \neq x$. 
To prove (2), consider an element $\operatorname{sts}^{-1}, t \in G(x)$. Then we have $\left(s t s^{-1}\right)(s(x))=s t(x)$. Since $t \in G(x), t(x) \neq x$. Since $s$ is an automorphism $s(t(x)) \neq s(x)$. Hence we have $\left(s t s^{-1}\right)(s(x)) \neq s(x)$. So $s t s^{-1} \in G(s(x))$.

Conversely if $\sigma \in G(s(x))$ we have $\sigma(s(x)) \neq s(x)$. So $s^{-1} \sigma s(x) \neq x$, so that $s^{-1} \sigma s \in G(x)$. Now $\sigma=s\left(s^{-1} \sigma s\right) s^{-1} \in s G(x) s^{-1}$. Hence (2) follows.

PROPOSITION 2.7. Let $E$ be an extension fiezd of a fiezd $K$ and let $E$ be Galois over $K$ with $G$ as the Galois group of $E$ over $K$. Let the topology $J$ be introduced in $G$ as in Definition 2.5. Then

(1) for $(G, J)$ translations are homeomorphisms,

(2) for $(G, J)$ inverse is a homeomorphism,

(3) $(G, J)$ has a sub-base of open sets consisting of sets of the form $\sigma G(y), \quad \sigma \in G, y \in E$.

Proof (1). It is enough to show that translations are continuous since the inverse of a translation is also a translation. To show that a map is continuous it is enough to show that the pre-image of a sub-basic open set is an open set. Consider for instance a translation $p \rightarrow \sigma p$. Then the pre-image of $s G(x) t$ is $\sigma^{-1}(s G(x) t)=\left(\sigma^{-1} s\right) G(x) t$. This is a sub-basic open set. Hence the map $p \rightarrow \sigma p$ is continuous. Similarly the map $p \rightarrow p \sigma$ is continuous. Hence (1) follows.

(2). Here it is enough to show that the map $p \rightarrow p^{-1}$ is continuous. The pre-image of $s G(x) t$ when $G(x) \neq \varnothing$ under the map $p \rightarrow p^{-1}$ is $t^{-1}(G(x))^{-1} s^{-1}$. But $(G(x))^{-1}=G(x)$. Hence $t^{-1}(G(x))^{-1} s^{-1}=t^{-1} G(x) s^{-1}$ which is a sub-basic open set.

(3). By Definition 2.5, $J$ has a sub-base consisting of sets of the form $s G(x) t$. Now $s G(x) t=(s t) t^{-1} G(x) t=(s t) G\left(t^{-1}(x)\right)$ by Proposition 2.6 and $(s t) G\left(t^{-1}(x)\right)$ is of the form $\sigma G(y)$.

THEOREM 2.8. Let $E$ be an extension field of a field $K$. Let 
further, for each intermediate field $F$ of $E / K, E$ be Galois over $F$. Let $G$ be the Galois group of $E$ over $K$. Let the topology $J$ be introduced on $G$ according to Definition 2.5. Then

(1) there exists a one-one Gazois correspondence between alz intermediate fields of $E / K$ and all J-closed subgroups of $G$; (a $J$-closed subgroup is a subgroup of $G$ which is a closed set for the topology $J$;)

(2) for $(G, J)$ translations and inverse are homeomorphisms.

Proof (1). If $F$ is an intermediate field of $E / K$ we let $g(F)=\{\sigma \in G \mid \sigma(x)=x$ for each $x \in F\}$; that is $g(F)$ is the Galois group of $E$ over $F, g(E)$ is a subgroup of $G$. By hypothesis, since $E$ is Galois over $F$, the fixed field of $g(F)$ is $F$. Hence $I(g(F))=F$. Hence $g(F)$ is a Galois closed subgroup of $G$. If $H$ is a Galois closed subgroup of $G$ then we have $g(I(H))=H$. Hence it follows that there exists a one-one Galois correspondence between all intermediate fields of $E / K$ and all Galois closed subgroups of $G$. So it is enough to show that a subgroup $H$ of $G$ is Galois closed if and only if $H$ is $J$-closed. But this is asserted by the proof of Theorem 3.1 of §3.

(2) is part of the Proposition 2.7.

THEOREM 2.9. Let $E$ be a Dedekind extension of $K$ and let $G$ be the Galois group of $E$ over $K$. Let the topology $J$ be introduced on $G$ according to Definition 2.5. If $E$ is algebraic over $K$ (that is if $E$ is an algebraic sepaxable normal extension of $K$ ) then $J$ coincides with the KruzL topology on $G$ and conversely if $J$ coincides with the KruzL topology on $G$ then $E$ must be algebraic separable normal over $K$.

Proof. Suppose $E$ is algebraic over $K$. Then the Krull topology on $G$ makes $G$ into a topological group and has a basis at identity consisting of subgroups $G \sim G(x), x \in E \sim K$. Now $G \sim G(x)$ is a Galois closed subgroup and hence is closed for the Krull topology. Hence $G(x)$ is open for the Krull topology. Hence also $s G(x) t$ is open for the Krull topology. Thus the Krull topology is finer than $J$. But for $(G, J)$ translations are homeomorphisms, and Galois closed subgroups are $J$-closed. We have proved in [9] that Krull topology is the coarsest 
topology on $G$ such that translations are homeomorphisms and Galois closed subgroups are topologically closed. Hence it follows that in this case $J$ coincides with the Krull topology.

Converse. Suppose $J$ coincides with the Krufl topology on $G$. We show that $E$ must be algebraic separable normal over $K$.

The Krull topology on $G$ is given by the convergence of nets as follows: a net $\sigma_{d}$ converges to an element $\sigma$ in $G$ if given any $x \in E$, there is a stage $d_{0}$ such that for all $d \geq d_{0}, \sigma_{d}(x)=\sigma(x)$. With this Krull topology $G$ becomes a topological group and then by Theorem 2.8, we have $(E, K ; G, J)$ a topological Galois system in the sense of [9] or [7]. Then Theorem 3 of [9] or the theorem of [7] shows that $E$ must be algebraic separable normal over $K$.

THEOREM 2.10. Let $E$ be a Dedekind extension of $K$ and let $G$ be the Galois group of $E$ over $K$. Let the topology $J$ be introduced on $G$ according to Definition 2.5. If $E$ is of finite transcendence degree over $K$ then $(G, J)$ is a compact space.

Proof. Let $F$ be any intermediate field of $E / K$ and let $\bar{F}$ be the relative algebraic closure of $F$ in $E$. Let $\sigma$ be any automorphism of $\bar{F}$ over $F$. Now $E$ is of finite transcendence degree over $F$ also. Let $x_{1}, \ldots, x_{r}$ be a transcendence base of $E$ over $F, x_{1}, \ldots, x_{r}$ are algebraically independent over $\bar{F}$ also. Consider the sub-field $F\left(x_{1}, \ldots, x_{p}\right)$. Then $\sigma$ can easily be extended to an automorphism $\bar{\sigma}$ of $\bar{F}\left(x_{1}, \ldots, x_{p}\right)$ over $E\left(x_{1}, \ldots, x_{p}\right)$. By hypothesis $E$ is Galois over $F\left(x_{1}, \ldots, x_{r}\right)$ and it is algebraic over $F\left(x_{1}, \ldots, x_{\gamma_{0}}\right)$. Hence $E$ is an algebraic separable normal extension of $F\left(x_{1}, \ldots, x_{p}\right)$. Now $F\left(x_{1}, \ldots, x_{r}\right) \subset \bar{F}\left(x_{1}, \ldots, x_{r}\right) \subset E$ and $\bar{\sigma}$ is an automorphism of $\bar{F}\left(x_{1}, \ldots, x_{p}\right)$ over $F\left(x_{1}, \ldots, x_{p}\right)$. Hence $\bar{\sigma}$ can be extended to an automorphism $\sigma_{1}$ of $E$ over $F\left(x_{1}, \ldots, x_{p}\right)$. Thus the automorphism $\sigma$ of $\bar{F}$ over $F$ has been extended to an automorphism $\sigma_{1}$ of $E$ over $F$. Now the assertion follows from Theorem 4.3 of $\S 4$.

PROPOSITION 2.11. Let $E$ be an algebraically closed extension fiezd 
of a fiezd $K$ of characteristic zero with infinite transcendence degree over $K$. Let $G$ be the Galois group of $E$ over $K$ and let the topology $J$ be introduced in $G$ according to Definition 2.5. Then

(a) $E$ is a Dedekind extension of $K$, and

(b) the topology $J$ is not compact.

Proof (a). Suppose $F$ is any intermediate field of $E / K$ and $x \in E \sim F$. If $x$ is transcendental over $F$ we can have a transcendence base $B$ of $E / F$ such that $x^{2} \in B$. Then $E$ is algebraic separable normal over $F(B)$ and hence we can find an automorphism fixing each element of $F(B)$ and moving $x$. If $x$ is algebraic over $F$ and $B$ is any transcendence base of $E / F$ then $x \notin F(B)$ and hence by a similar argument as above, there is an automorphism of $E$ over $F$ moving $x$. Hence $E$ is Galois over $F$ and hence $E$ is a Dedekind extension of $K$.

(b). Let $B$ be a transcendence base of $E$ over $K$. Write $B=B_{1} \cup B_{2}$, where $B_{1} \cap B_{2}=\varnothing$ and $B_{1}=\left\{x_{1}, x_{2}, \ldots\right\}$ a countably infinite set. Let $H$ be the Galois group of $E$ over $K\left(B_{2}\right)$ and for each $i, H_{i}$ be the Galois group of $E$ over $K\left(B_{2}, x_{i}\right)$. We note that $H_{i} \subset H$ and $H_{i}$ and $H$ are $J$-closed subgroups since they are Galois closed subgroups. Since $E$ is an algebraically closed extension field of $K\left(B_{2}\right)$, to each $i$ we can have an automorphism $\sigma_{i}$ of $E / K$ such that $\sigma_{i}\left(x_{i}\right)=x_{i+1}, \sigma_{i}\left(x_{i+1}\right)=x_{i}, \sigma_{i}\left(x_{j}\right)=x_{j}$ if $j \neq i, i+1$ and $\sigma_{i}$ fixes each element of $B_{2}$. Since translations are homeomorphisms for $J$, $\sigma_{i} H_{i}$ is a closed set. Now we consider the collection of closed sets $\left\{\sigma_{i} H_{i}\right\}, i=1,2, \ldots$.

(i) This collection has the finite intersection property: for consider $\sigma_{1} H_{1} \cap \ldots \cap \sigma_{n}{ }_{n}$. The mapping $x_{1} \rightarrow x_{2}$, $x_{2} \rightarrow x_{3}, \ldots, x_{n} \rightarrow x_{n+1}, x_{n+1} \rightarrow x_{1}, x_{n+j}+x_{n+j}$ for $j=2,3, \ldots, y \rightarrow y$ for every $y \in B_{2}$, yields an automorphism of $K(B)$ over $K$ and since $E$ is an algebraically closed extension of $K(B)$ this can be extended to an automorphism $\sigma$ of $E$ over $K$. We assert that $\sigma \in \sigma_{1} H_{1} \cap \ldots \cap \sigma_{n} H_{n}$. Consider $H_{i}, 1 \leq i \leq n$. Consider the 
automorphism $\sigma_{i}^{-1} \sigma$. Since $\sigma_{i}$ and $\sigma$ fix each element of $B_{2}$ we have $\sigma_{i}^{-1} \sigma$ also fixes each element of $B_{2}$. Also $\sigma_{i}^{-1} \sigma\left(x_{i}\right)=\sigma_{i}^{-1}\left(x_{i+1}\right)=x_{i}$. Hence we get that $\sigma_{i}^{-1} \sigma \in H_{i}$. Hence $\sigma \in \sigma_{i} H_{i}$.

(ii) $\prod_{I}^{\infty} \sigma_{i} H_{i}=\emptyset$. For suppose an automorphism $s \in \prod_{1}^{\infty} \sigma_{i} H_{i}$. Then $s \in H$ since each $\sigma_{i} H_{i} \subset H$. So $s$ is an automorphism of $E$ over $K\left(B_{2}\right)$. Also for each $i$ we have $s \in \sigma_{i} H_{i}$. Hence $\sigma_{i}^{-1} s \in H_{i}$, that is $\sigma_{i}\left(x_{i}\right)=s\left(x_{i}\right)$. So $s\left(x_{i}\right)=x_{i+1}$ for every $i$. Under the automorphism $s$ of $E$ over $K\left(B_{2}\right)$ let $\bar{x}_{1}$ be the pre-image of $x_{1}$. Then $\bar{x}_{1}$ is algebraic over $K(B)$. So there is an algebraic relation connecting $\bar{x}_{1}$ and elements of $B$ with coefficients in $K$. Now $s\left(\bar{x}_{1}\right)=x_{1}$ and $s(B) \subset B$. Applying $s$ we get an algebraic relation connecting elements of $B$. This is a contradiction. Hence $\prod_{1}^{\infty} \sigma_{i} H_{i}=\varphi$.

Thus (i) and (ii) show that $J$ is not compact since there is a family of closed sets with finite intersection property but intersection of all the members of the family is empty.

THEOREM 2.12. Let $E$ be an algebraically closed extension field of a field $K$ with infinite transcendence degree. Let $G$ be the Galois group of $E$ over $K$. Then there cannot exist a compact topology $T$ on $G$ such that translations are homeomorphisms and such that there is a one-one Galois correspondence between all the intermediate fields of $E / K$ and alz the T-closed subgroups of $G$.

Proof. Suppose there is a topology $T$ on $G$ satisfying the conditions of the theorem. It is easily shown that $E$ must be of characteristic zero [9]. Then Theorem 4.1 of $\$ 4$ shows that $T$ is finer than $J$. But $T$ is compact. Hence we get that $J$ is compact. But this contradicts Theorem 2.11. Hence the theorem follows.

3.

In this section we prove the following theorem. 
THEOREM 3.1. Let $E$ be any extension field of a field $K$ and let $E$ be Galois over $K$. Let further $G$ be the Galois group of $E$ over $K$. Then there exists a topology $T$ on $G$ such that:

(1) the Galois closed subgroups of $G$ are precisely the subgroups which are closed subsets under the topology $T$;

(2) for $(G, T)$ transzations and inverse are homeomorphisms.

Proof. Let the topology $J$ be introduced on $G$ according to Definition 2.5. Then by Proposition 2.7, the condition 2 of Theorem 3.1 is satisfied by $(G, J)$. We have only to show that the Galois closed subgroups of $G$ are precisely the $J$-closed subgroups of $G$.

Let $H$ be a Galois closed subgroup of $G$. Let $s$ be an element of $G$ not belonging to $H$. Since $H$ is Galois closed

$$
H=\{t \in G \mid t x=x \text { for every } x \in I(H) \text {, the fixed field of } H\} \text {. }
$$

Hence there is an element $x \in I(H)$ such that $s(x) \neq x$. Consider now $G(x)$. Then $G(x)$ is an open set under $J$ and $s \in G(x)$. Since $x \in I(H)$, no element of $H$ belongs to $G(x)$. Hence to each $s \in G \sim H$ there is a $J$-open set containing $x$ and completely contained in $G \sim H$. Hence $G \sim H$ is open and so $H$ is closed under $J$.

Conversely let now $H$ be a subgroup of $G$ which is a closed set under the topology $J$. We show $H$ is a Galois closed subgroup of $G$. Let $I(H)$ be the fixed field of $H$. Let $\sigma$ be an element of $G$ leaving each element of $I(H)$ fixed. We have to show that $\sigma \in H$. We assert now that every neighbourhood of $\sigma$ intersects $H$. It is enough to show that every basic open set containing $\sigma$ intersects $H$. By Proposition 2.7, we can take a basic open set containing $\sigma$ to be of the form

$$
s_{1} G\left(x_{1}\right) \cap s_{2} G\left(x_{2}\right) \cap \ldots \cap s_{n} G\left(x_{n}\right) \text {. }
$$

Case 1. Each of the $x_{1}, \ldots, x_{n}$ is algebraic over $I(H)$. Then each $x_{i}$ has only a finite number of distinct images $x_{i 1}, x_{i 2}, \ldots, x_{i r}$ by $H$. If we consider the elementary symmetric functions on the $x_{i l}, \ldots, x_{i r_{i}}$, all these are left fixed by each element of $H$ and hence they belong to $I(H)$. Hence the polynomial $\left(x-x_{i l}\right) \ldots\left(x_{i r}\right)$ is an 
irreducible polynomial for $x$ over $I(H)$. Thus it follows that $x_{i}$ is separable algebraic over $I(H)$. Hence the sub-field $I(H)\left(x_{1}, \ldots, x_{n}\right)$ is contained in a finite separable normal extension

$$
F=I(H)\left(x_{11}, \ldots, x_{1 r_{1}}, x_{2 I}, \ldots, x_{2 r_{2}}, \ldots, x_{n I}, \ldots, x_{n r_{n}}\right)
$$

of $I(H)$ and also $F \subset E$. Since any finite separable extension is simple we have $F=I(H)(\theta)$. Now each automorphism of $E$ over $I(H)$ induces an automorphism of $F$ over $I(H)$ since $F$ is finite separable normal over $I(H)$. As before, if $\theta_{1}=\theta, \theta_{2}, \ldots, \theta_{m}$ is the complete set of distinct images of $\theta$ under $H$ then $\left(x-\theta_{1}\right) \ldots\left(x-\theta_{m}\right)$ is an irreducible polynomial for $\theta$ over $I(H)$ and every automorphism of $F$ over $I(H)$ has to take $\theta$ to some $\theta_{i}$. Hence $\theta \rightarrow \theta_{i} \quad(i=1,2, \ldots, n)$ give the complete set of automorphisms of $F$ over $I(H)$. Also each element of $B$ induces an automorphism of $F$ over $I(H)$. Further, given $i$ there is an element of $H$ taking $\theta$ to $\theta_{i}$. Now $\sigma$ also induces an automorphism of $F$ over $I(H)$ since $\sigma$ leaves each element of $I(H)$ fixed. But $H$ induces the complete set of automorphisms of $F$ over $I(H)$. We get that there exists an element $h \in H$ such that $\sigma$ and $h$ induce the same automorphism of $F$ over $I(H)$. Since $x_{i} \in F$ we have $\sigma\left(x_{i}\right)=h\left(x_{i}\right)$. Hence also $s_{i}^{-1}\left(\sigma\left(x_{i}\right)\right)=s_{i}^{-1}\left(h\left(x_{i}\right)\right)$. So $\left(s_{i}^{-1} \sigma\right)\left(x_{i}\right)=\left(s_{i}^{-1} h\right)\left(x_{i}\right)$. Since $\sigma \in s_{i} G\left(x_{i}\right)$ we have $s_{i}^{-1} \sigma \in G\left(x_{i}\right)$ and hence $\left(s_{i}^{-1} \sigma\right)\left(x_{i}\right) \neq x_{i}$. So $s_{i}^{-1} h\left(x_{i}\right) \neq x_{i}$ and hence $s_{i}^{-1} h \in G\left(x_{i}\right)$ and so $h \in s_{i} G\left(x_{i}\right)$. Hence it follows that $h \in s_{1} G\left(x_{1}\right) \cap \ldots \cap s_{n} G\left(x_{n}\right)$. Hence this open set $s_{1} G\left(x_{1}\right) \cap \ldots \cap s_{n} G\left(x_{n}\right)$ intersects $H$.

Case 2. At least one of the $x_{i}$ is transcendental over $I(H)$. Let if possible $s_{1} G\left(x_{1}\right) \cap \ldots \cap s_{n} G\left(x_{n}\right) \cap H=\emptyset$. We will get a contradiction. Let $H_{i}=\left\{s \in G \mid s\left(x_{i}\right)=x_{i}\right\}$, for $i=1,2, \ldots, n$. Then $H_{i}$ is a subgroup of $G$. We also have $G \sim s_{i} G\left(x_{i}\right)=s_{i} H_{i}$. We now have $H \subset s_{1} H_{1} \cup \ldots \cup s_{n}{ }_{n}$. If for some $i$ it happens that $H \cap s_{i} H_{i}$ 
is contained in the set union $\underset{j \neq i}{U} s_{j}{ }_{j}$, then this implies that any element belonging to $H \cap\left(\bigcap_{j \neq i} s_{j} G\left(x_{j}\right)\right)$ belongs to $s_{i} G\left(x_{i}\right)$ also. Hence it is enough for us to show that $H \cap\left(\bigcap_{j \neq i} s_{j} G\left(x_{j}\right)\right) \neq \emptyset$; that is we could drop $s_{i} G\left(x_{i}\right)$ out of the picture. Proceeding successively thus we arrive at a stage where no $s_{i} G\left(x_{i}\right)$ can be omitted further. If at that stage all the $x_{i}$ that occur are algebraic over $I(H)$ then Case 1 completes the proof.

Hence we can assume $H \subset s_{1} H_{1} \cup \ldots \cup s_{n}{ }_{n}$ where no $s_{i} H_{i}$ can be omitted and that at least one of the $x_{i}$ is transcendental over $I(H)$. Let, for definiteness, $x_{1}$ be transcendental over $I(H)$. Then the number of distinct images of $x_{1}$ under $H$ is infinite (since otherwise $x_{1}$ will be algebraic over $I(H)$ using the trick of Case 1). Now by Lemma $1 . I$ we have that if we put $H^{I}=H_{1} \cap \ldots \cap H_{n}$ then $H \subset U_{l}^{p} t_{k} H^{I}$, a set union of a finite number of cosets of $H^{1}$. Now each element of $H^{1}$ fixes $x_{1}$. If $h \in H$ then $h=t_{i} h_{1}, h_{1} \in H$, for some $i$. Hence $h\left(x_{1}\right)=t_{i} h_{1}\left(x_{1}\right)=t_{i}\left(x_{1}\right)$. Hence the number of distinct 1mages of $x_{1}$ under $H$ is at most $p$. This is a contradiction.

Hence we have that each basic open set containing $\sigma$ intersects $H$. Hence $\sigma$ belongs to the closure of $H$. But $H$ is closed. Hence $\sigma \in H$. So every automorphism of $E$ over $I(H)$ belongs to $H$. Hence $H$ is Galois closed.

This establishes Theorem 3.1 with $T=J$.

Theorem 3.1 allows for an algebraic interpretation.

THEOREM 3.2. Let $E$ be a Galois extension of a fiezd $K$ and let $G$ be the Galois group of $E$ over $K$. Then a subgroup $H$ of $G$ is Galois closed if and only if the following condition is satisfied:

Given any $\sigma \notin H$ there exists a finite number of elements $s_{1}, s_{2}, \ldots, s_{n}$ of $G$ such that for each $i s_{i}\left(x_{i}\right) \neq \sigma\left(x_{i}\right)$, but 
given any $h \in H$ there is an $i$ such that $h\left(x_{i}\right)=s_{i}\left(x_{i}\right)$.

4.

Throughout this section:

Let $E$ be an extension field of a field $K$ and let $E$ be Galois over $K$. Let $G$ be the Galois group of $E$ over $K$. Let the topology $J$ be introduced in $G$ according to Definition 2.5.

PROPOSITION 4.1 .

(a) $J$ is the coarsest topology on $G$ such that Galois closed subgroups are topologically closed and translations are homeomorphisms;

(b) $(G, J)$ is a $T_{1}$-space.

Proof (a). By the proofs of Theorem 3.1 and Proposition 2.7 Galois closed subgroups are $J$-closed and translations are homeomorphisms for $(G, J)$.

Let now $T$ be any topology on $G$ such that Galois closed subgroups are $T$-closed and translations are homeomorphisms. We show $T$ is finer than $J$; that is every $J$-open set is $T$-open. It is enough to show that any $s G(x) t$ is $T$-open whenever $G(x) \neq \emptyset$. If we let $H_{x}=\{\sigma \in G \mid \sigma(x)=x\}$ then $H_{x}$ is a Galois closed subgroup and hence is closed under $T$. Then $G \sim H_{x}$ is T-open. But $G(x)=G \sim H_{x}$. Hence $G(x)$ is $T$-open. Since translations are homeomorphisms for $(G, T)$ we have first that $s G(x)$ is $T$-open and then $s G(x) t$ is $T$-open. This establishes (a).

(b). Since the identity $\{e\}$ is a Galois closed subgroup we have that the one-point set $\{e\}$ is closed under $J$. Since translations are homeomorphisms for $(G, J)$ we get that for each $\sigma \in G$, the set $\{\sigma\}=\sigma\{e\}$ is $J$-closed and hence $(G, J)$ is a $T_{1}$-space.

THEOREM 4.2. Let $E$ be a Dedekind extension of $K$. Then the topology $J$ is Hausdorff if and only if $E$ is algebraic separable normal over $K$.

Proof. Suppose $E$ is algebraic separable normal over $K$. Then $J$ 
coincides with the Krull topology on $G$ by Theorem 2.9 and with the Krull topology it is well known that $G$ is a Hausdorff topological group [1, Chapitres 4 et $5 ; 2]$.

Conversely, suppose now $J$ is Hausdorff. Already by Proposition 2.7 translations are homeomorphisms for $(G, J)$ and by Theorem 2.8, $(E, K, G, J)$ is a topological Galois system in the sense of [9]. Then Theorem 4 of [9] completes the proof.

THEOREM 4.3. Let $E$ be any extension of finite transcendence degree over $K$. Then the topology $J$ is compact if and only if the following condition is satisfied. Whenever $F$ is an intermediate field of $E / K$ such that $E$ is Galois over $F$ and $\vec{F}$ denotes the relative algebraic closure of $F$ in $E$ (that is, $\bar{F}=\{x \in E \mid x$ is algebraic over $F\}$, then any automorphism of $\bar{F}$ over $F$ can be extended to an automorphism of $E$ over $F$.

Proof. Suppose $J$ is compact. Let $F$ be an intermediate field such that $E$ is Galois over $F$ and let $\bar{F}$ be the relative algebraic closure of $F$ in $E$ and let $\sigma$ be an automorphism of $\bar{F}$ over $F$. We show that $\sigma$ is extendible to an automorphism of $E$ over $F$.

Since $E$ is Galois over $F, F$ is the fixed field of the Galois group of $E$ over $F$ and each automorphism of $E$ over $F$ leaves $\bar{F}$ set-wise invariant so that it is easy to show that (using the trick of the Proof of Theorem 3.1) that $\bar{F}$ is algebraic separable normal over $F$.

If $x$ is any element of $\bar{F}$, then using the trick of the Proof of Theorem 3.1, we can find a $\theta \in \bar{F}$ such that $F(x) \subset F(\theta)$ and $F(\theta)$ is finite separable normal over $F$ and the Galois group of $E$ over $F$ induces the full group of automorphisms of $F(\theta)$ over $F$. We note here that if $F_{2}$ is any field such that $F \subset F_{2} \subset \bar{F}$ and $F_{2}$ is finite separable normal over $F$ then the Galois group of $E$ over $F$ induces the full group of automorphisms of $F_{2}$ over $F$.

Hence we can write $\bar{F}=F(B)$ where $B \subset \bar{F}$ is a generating set for $\bar{F}$ over $F$ such that for each $x \in B, F(x)$ is finite separable normal over $F$. For $x \in B$, let

$$
H_{x}=\{t \in G \mid t \text { leaves each element of } F(x) \text { fixed }\} \text {. }
$$


Then $H_{x}$ is a Galois closed subgroup of $G$ and hence is a $J$-closed subset. Now there exists an automorphism $s_{x}$ of $E$ over $F$ such that $s_{x}$ and $\sigma$ induce the same automorphisms of $F(x)$ over $F$. (Note that since $F(x)$ is finite separable normal over $F$ and $\sigma$ is an automorphism of $\bar{F}$ over $F, \sigma$ induces an automorphism of $F(x)$ over $F$.) since translations are homeomorphisms for $(G, J), s_{x}^{H} x$ is a J-closed set. Consider now the collection $\left\{s_{x}{ }_{x}\right\}_{x \in B}$ of closed subsets of $G$. We assert that this family has the finite intersection property. Consider $s_{x_{1}}{ }^{H} x_{1}, \ldots, s_{x_{n}}{ }^{H} x_{n}$. Consider the subfield $F\left(x_{1}, \ldots, x_{n}\right)$. This is a finite separable normal extension of $F$ since each $F\left(x_{i}\right) / F$ is finite separable normal. Then there exists an element $h$ in the Galois group of $E$ over $F$ such that $h$ and $\sigma$ induce the same automorphism of $F\left(x_{1}, \ldots, x_{n}\right)$ over $F$. We assert that $h \in s_{x_{1}}{ }^{H} x_{1} \cap \ldots \cap s_{x_{n}}{ }^{H} x_{n}$. For we have $h\left(x_{i}\right)=\sigma\left(x_{i}\right)=s_{x_{i}}\left(x_{i}\right)$ and so $\left(s_{x_{i}}^{-1}\right)\left(x_{i}\right)=x_{i}$. Already $s_{x_{i}}$ and $h$ are automorphisms of $E$ over $F$. Hence we get $s_{x_{i}}^{-1} h \in{ }_{x_{i}}$, that is $h \in s_{x_{i}}{ }^{H} x_{i}$. Since $J$ is compact there is an element $s$ such that $s \in s_{x}{ }^{H} x$ for every $x \in B$. Observe that for each $x_{i}$, $\sigma\left(x_{i}\right)=s_{x_{i}}\left(x_{i}\right)=s\left(x_{i}\right)$. Now this $s$ is an automorphism of $E$ over $F$ and we assert that this $s$ extends $\sigma$. For if $y \in F$ then $y \in F\left(x_{1}, \ldots, x_{n}\right)$ for some $x_{1}, \ldots, x_{n}$ in $B$. Hence $y=p\left(x_{1}, \ldots, x_{n}\right)$ a polynomial in $x_{1}, \ldots, x_{n}$ with coefficients in $F$. Then

$$
s(y)=p\left(s\left(x_{1}\right), \ldots, s\left(x_{n}\right)\right)=p\left(\sigma\left(x_{1}\right), \ldots, \sigma\left(x_{n}\right)\right)=\sigma(y)
$$

since $s\left(x_{i}\right)=\sigma\left(x_{i}\right)$ for each $x_{i}$. Hence $s(y)=\sigma(y)$. This completes the necessity.

Suppose the condition is satisfied. We show that $J$ is compact. By Alexander's Theorem it is enough to show that any open cover $U$ of $G$ by 
non-empty sets of the form $s G(x), s \in G, x \in E$ has a finite subcover since sets of the form $s G(x)$ form a sub-base. Suppose there are two members in the cover of the form $s_{1} G(x)$ and $s_{2} G(x)$ with $s_{1} G(x) \neq s_{2} G(x)$. Then we first have $s_{1}(x) \neq s_{2}(x)$. Otherwise if $s_{1}(x)=s_{2}(x)$ and $\sigma \in s_{1} G(x)$ then $\sigma(x) \neq s_{1}(x)$ and hence $\sigma(x) \neq s_{2}(x)$ and so $\sigma \in s_{2} G(x)$. So $s_{1} G(x) \subset s_{2} G(x)$ and similarly $s_{2} G(x) \subset s_{1} G(x)$ and hence $s_{1} G(x)=s_{2} G(x)$, a contradiction. Now we claim that $G=s_{1} G(x) \cup s_{2} G(x)$. For if $\sigma \in G$ and $\sigma$ is not an element of the right-hand side then $s_{1}^{-1} \sigma \notin G(x)$, and $s_{2}^{-1} \sigma \notin G(x)$ and hence we have $s_{1}(x)=\sigma(x)=s_{2}(x)$ a contradiction.

Hence we can suppose that our open cover $U$ consists of non-empty sets of the form $s_{x} G(x), s_{x} \in G$ and $x \in E$.

Suppose this cover has no finite sub-cover. We will get a contradicion. Consider the family $\left\{s_{x}{ }_{x}\right\}$ where ${ }_{x}=\{\sigma \in G \mid \sigma(x)=x\}, s_{x}{ }_{x}=G \sim s_{x} G(x)$. Hence we have a family of closed sets $s_{x}{ }_{x}$. This family has now the finite intersection property. We have only to show that ${ }_{s_{x} G(x) \in U} s_{x}{ }^{H} x \neq \emptyset$. Consider the set of all elements $x$ such that $s_{x} G(x) \in U$. Since $E / K$ has finite transcendence degree, we can find a finite number of elements $x_{1}, x_{2}, \ldots, x_{r}$ here such that every other $x$ here is algebraic over $K\left(x_{1}, \ldots, x_{p}\right)$. There exists an element $\sigma_{1} \in s_{x_{1}}{ }^{H} x_{1} \cap \cdots \cap s_{x_{p}}{ }^{H} x_{r}$. Since $s \rightarrow \sigma_{1} s$ is a homeomorphism of $(G, J)$ it is enough to consider the family of closed sets $\left\{\left(\sigma_{I}^{-1} s_{x}\right)_{x}\right\}$ with finite intersection property and show that $\cap\left(\sigma_{1}^{-1} s_{x}\right) H_{x} \neq \emptyset$. In this case for each $i=1,2, \ldots, r$, any element of $\left(\sigma_{1}^{-1} s_{x_{i}}{ }^{H}{ }_{x_{i}}\right.$ leaves $x_{i}$ fixed.

Hence we can suppose that our family $\left\{s_{x}{ }_{x}\right\}$ is such that for $x_{1}, \ldots, x_{r}, s_{x_{i}}\left(x_{i}\right)=x_{i}$. Let us put now $F=k\left(x_{1}, \ldots, x_{r}\right)$. Let 
$F_{1}$ be the smallest Galois closed field containing $F$, that is $F_{1}=\{x \in E \mid \sigma(x)=x$ whenever $\sigma$ fixes each element of $F\}$. Then each $x$ such that $s_{x} G(x) \in U$ has the property that $x \in \bar{F}_{1}$, the algebraic closure of $F_{1}$ in $E$. Consider now the correspondence $x_{1} \rightarrow x_{1}, x_{2} \rightarrow x_{2}, \ldots, x_{r}+x_{r}$ and $x \rightarrow s_{x}(x)$, whenever $x$ is such that $s_{x} G(x) \in U$. This correspondence yields an isomorphism of the field $E_{1}\left(\{x\} s_{x} G(x) \in U\right)$ containing $F_{1}$ into $\bar{F}_{1}$ fixing each element of $F_{1}$ since for any finite number of elements $x_{1}, \ldots, x_{r}, x_{\alpha_{1}}, \ldots, x_{\alpha_{n}}$ there exists an automorphism of $E / K$ belonging to

$$
s_{x_{1}}{ }^{H} x_{1} \cap s_{x_{2}}{ }^{H} x_{2} \cap \ldots \cap s_{x_{p}}{ }^{H} x_{x} \cap s_{x_{\alpha_{1}}}{ }^{H}{ }_{x_{\alpha_{1}}} \cap \ldots \cap s_{x_{\alpha_{n}}}{ }^{H} x_{\alpha_{n}} \text {. }
$$

Since $E$ is Galois over $F_{1}$, we can easily show that $\bar{F}_{1}$ is algebraic separable normal over $F_{1}$; and now we have an isomorphism over $F_{1}$ of an intermediate field of $\bar{F}_{1} / F_{1}$ into $\bar{F}_{1}$. This, as is well known, can be extended to an automorphism of $\bar{F}_{1} / F_{1}$. Now $E$ is Galois over $F_{1}$ and so by hypothesis this can be extended to an automorphism $\sigma_{2}$ of $E / F_{1}$. For each $x$ we have $\sigma_{2}(x)=s_{x}(x)$. Hence $\sigma_{2} \in s_{x}^{H} x$. Hence $\sigma_{2} \in \Pi s_{x}^{H}{ }_{x}$

The sufficiency now follows.

COROLLARY 4.4. Let $E$ be a finitely generated extension of $K$. Then $J$ is compact.

Proof. Since $E$ is finitely generated over $K, E$ is of finite transcendence degree over $K$. Also if $\bar{F}$ is an intermediate field over which $E$ is Galois then $\bar{F}$ is finite over $F$ and hence it follows that the Galois group of $E$ over $F$ induces the full group of automorphisms of $\bar{F}$ over $F$. Now the result follows by Theorem 4.3 .

COROLLARY 4.5. If $E$ is a pure transcendental extension of finite transcendence degree then $J$ is compact.

Proof. Follows from Corollary 4.4.

COROLLARY 4.6. Suppose $K$ is algebraically closed in $E$ and $E$ 
has transcendence degree one over $K$. Then the topology $J$ is compact.

Proof. For $K, \bar{K}=K$ and hence the condition of Theorem 4.3 is satisfied easily. If $F$ is an intermediate field and $F \neq K$ then because $E / K$ is of transcendence degree one we get $\bar{F}=E$ itself and hence again the condition of Theorem 4.3 is satisfied. Hence the corollary follows by Theorem 4.3.

PROPOSITION 4.7. If $E=K(x)$, a simple transcendental extension, then $J$ is the minimal $T_{1}$-topology on $G$, and in this case $J$ is connected and compact.

Proof. For in this case for any $y \in E \sim K, G \sim G(y)$ is a finite set since $K(x)$ is a finite extension of $K(y)$ and hence it follows that for any basic open set its complement is finite. Hence $J$ is coarser than the minimal $T_{1}$-topology. But $J$ is already $T_{1}$. Hence it follows that $J$ coincides with the minimal $T_{1}$-topology; and it is well known that the minimal $T_{1}$-topology on an infinite set is both compact and connected.

\section{References}

[1] N. Bourbaki, Éléments de mathématique, Fascicules 28, 30. Algèbre commutative (Actualités Scientifiques et Industrielles, Nos. 1293, 1308. Hermann, Paris, 1961, 1964).

[2] Otto Endler, Teoma de Galois infinita (Notas de Matemática, No. 30. Fasciculo Pulibcado pelo Institute de Matemática Pure e Aplicado do Conselho Nacional de Pesquisas, Rio de Janeiro, 1965).

[3] Wolfgang Kru/I, "Galoissche Theorie der unendlichen algebraischen Erweiterungen", Math. Ann. 100 (1928), 687-698.

[4] B.H. Neumann, "Groups covered by finitely many cosets", Publ. Math. Debrecen 3 (1954), 227-242.

[5] T. Soundararajan, "A topology for extension fields and Galois theory", K. Nederi. Akad. Wetensch. Proc. Ser. A. 68 (1965), $136-140$. 
[6] T. Soundararajan, "Completeness of Galois theories", Indian J. Math. $8(1966), 11-14$.

[7] T. Soundararajan, "Completeness of Galois theories. II", J. Indian Math. Soc. (N.S.) 30 (1966), 69-72.

[8] T. Soundararajan, "A note on classical Galois theory", Math. Ann. 182 (1969), 275-280.

[9] T. Soundararajan, "Galois theory for general extension fields", $J$. reine angew. Math. 241 (1970), 49-63.

[10] T. Soundararajan and K. Venkatachaliengar, "Topologies for Galois groups of extension fields", J. reine angew. Math. 242 (1970), , $1-7$.

[11] M. Venkataraman and T. Soundararajan, "On the completeness of Galois theories", J. Austral. Math. Soc. 5 (1965), 374-379.

Department of Mathematics, Madurai University,

India. 